hep-th/9902026

\title{
$\widehat{U(1)} \times S \widehat{U(m)}$, Theory and $c=m W_{1+\infty}$ Minimal
}

\section{Models in the Hierarchical Quantum Hall Effect}

\author{
Marina HUERTA \\ Centro Atómico Bariloche and Instituto Balseiro, C. N. E. A. and \\ Universidad Nacional de Cuyo, 8400 - San Carlos de Bariloche, Río Negro, \\ Argentina
}

\begin{abstract}
Two classes of Conformal Field Theories have been proposed to describe the Hierarchical Quantum Hall Effect: the multi-component bosonic theory, characterized by the symmetry $\widehat{U(1)} \times S \widehat{U(m)_{1}}$ and the $W_{1+\infty}$ minimal models with central charge $c=m$. In spite of having the same spectrum of edge excitations, they manifest differences in the degeneracy of the states and in the quantum statistics, which call for a more detailed comparison between them. Here, we describe their detailed relation for the general case, $c=m$ and extend the methods previously published for $c \leq 3$. Specifically, we obtain the reduction in the number of degrees of freedom from the multi-component Abelian theory to the minimal models by decomposing the characters of the $\widehat{U(1)} \times S \widehat{U(m)}{ }_{1}$ representations into those of the $c=m W_{1+\infty}$ minimal models. Furthermore, we find the Hamiltonian whose renormalization group flow interpolates between the two models, having the $W_{1+\infty}$ minimal models as infra-red fixed point.
\end{abstract}

January 1999 


\section{Introduction}

The low energy edge excitations of incompressible quantum Hall fluids [1] are one-dimensional chiral waves which propagate on the boundary of the sample. These could be described by $(1+1)$ dimensional effective conformal field theories [2]. Moreover, this effective approach to describe the low energy physics, is in accordance with the high precision and universality found experimentally in the structure of the plateaus for the Hall conductivity.

The filling fractions of the more stable plateaus are given by the Jain series [3]:

$$
\nu=\frac{m}{m p+1},
$$

with $m=1,2,3, \ldots$ and $p=2,4,6, \ldots$ This corresponds to the majority of the filling fractions observed experimentally in the range $0<\nu<1$; therefore, its study and comprehension is very relevant in the Quantum Hall Effect problem. For the Laughlin case with filling fraction $\nu=\frac{1}{p+1}(m=1)$, the corresponding conformal theory is the Abelian chiral boson one, which describes the universal properties of the quasi-particle excitations; these are in agreement with the Laughlin's microscopic theory for the charge spectrum $Q$ and the quantum statistics $\theta / \pi$ 迎.

In contrast to the Laughlin case, general hierarchical Hall plateaus, whose filling fractions are given by the Jain formula (1) for general $m \neq 1$, are not well understood yet. At the moment, two classes of conformal field theories have been proposed. The first is the multi-component bosonic theory, characterized by the Abelian current algebra $\widehat{U(1)}{ }^{m}$ (more specifically $\widehat{U(1)} \times S \widehat{U(m)_{1}}$ ), which is a natural generalization of the Abelian case made by $m$ copies of the one component theory [5]. The second one is given by the minimal models of the $W_{1+\infty}$ symmetry algebra [6], based on the dynamical symmetry of classical incompressible fluids under area-preserving diffeomorphisms [7].

The spectra of these two types of theories are identical, but they differ in the degeneracy of their excitations. This can be tested by computing numerically the energy spectrum for the Hall states of a few electron system. This kind of study has shown a reduction in the number of degrees of freedom compared to the Abelian theory, which is in agreement with the prediction of the $W_{1+\infty}$ minimal models [8]. This result motivates our analysis of the 
general hierarchical problem.

Further important differences between the two theories are that the minimal models exist for the hierarchical plateaus only, and that they possess a single Abelian charge, rather than the $m$ charges of the Abelian multicomponent theory. Moreover, the minimal model quasi-particles possess a type of non-Abelian statistics: actually, they are labelled by the weights of the $S U(m)$ Lie algebra instead; the multi-component theories, on the contrary, they are characterized by the $(m-1)$ Abelian charges.

The connection between the two types of theories has been explicitly found in Ref. [9] for the $c=2$ and $c=3$ cases. By introducing the bosonic Fock space and by using the algebraic methods of representation theory, it is possible to show that the minimal models correspond to a sub-set of the states of the Abelian theory. This reduction in the number of degrees of freedom can be performed by adding an interaction term to the original Hamiltonian: this is relevant in the renormalization-group sense, such that the corresponding flow interpolates from the multi-component abelian to the minimal theories in the infra-red.

The purpose of this paper is to generalize these results to any $c=m>3$.

In section 2 we include a brief summary of the $c=2$ theory results. In

section 3 we present the decomposition of the $\widehat{U(1)} \times \widehat{S U(m)_{1}}$ characters into those of the $c=m W_{1+\infty}$ minimal models and the Hamiltonian that interpolates between the two theories..

\section{The $c=2$ case: Minimal model and two component Abelian theory}

The two component Abelian theories are built out of two chiral bosons $\varphi^{(i)}, i=1,2$, which are defined on the edge of the Hall sample, which we take as a disk parametrized by a radius $R$ and an angle $\theta$ [2]. The chiral currents $J^{(1)}$ and $J^{(2)}$ associated with these bosonic fields describe the edge excitations; their Fourier modes $\alpha_{n}^{(i)}, i=1,2$, satisfy the Abelian $\widehat{U(1)} \times \widehat{U(1)}$ current algebra

$$
\left[\alpha_{n}^{(i)}, \alpha_{n}^{(j)}\right]=\delta^{i j} n \delta_{n+m, 0}
$$


The associated generators of the Virasoro algebra satisfy

$$
\left[L_{n}^{(i)}, L_{n}^{(j)}\right]=\delta^{i j}\left\{(n-m) L_{n+m}^{(i)}+\frac{c}{12} n\left(n^{2}-1\right) \delta_{n+m, 0}\right\},
$$

with $c=1$, where $L_{n}^{(i)}$ are obtained from the Sugawara construction

$$
L_{n}^{(i)}=\frac{1}{2} \alpha_{0}^{(i)}+\sum_{n=0}^{\infty} \alpha_{-n}^{(i)} \alpha_{n}^{(i)} .
$$

The eigenvalues of $\alpha_{0}^{(i)}$ and $L_{0}^{(i)}$ characterize the highest weight representation of the Abelian algebra. Here, it is convenient to define $\alpha_{n}=\alpha_{n}^{(1)}+\alpha_{n}^{(2)}$ and $L_{n}=L_{n}^{(1)}+L_{n}^{(2)}$ with $c=2$ and the same algebra. The fractional statistics $\theta / \pi$ and the charge of the highest weight representations are, therefore, given by the eigenvalues of $\left(L_{0}^{(1)}+L_{0}^{(2)}\right)$ and $\left(\alpha_{0}^{(1)}+\alpha_{0}^{(2)}\right)$, respectively. It is

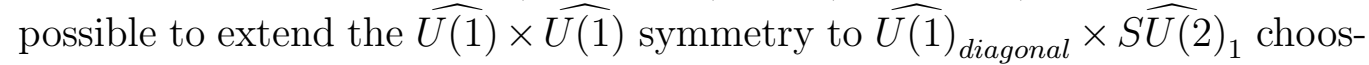
ing an appropriate set of representations closed under the fusion rules. This choice reproduces the Jain values of the filling fraction $\nu=2 / 5,2 / 3, \ldots$. More precisely, the $\widehat{U(1)} \times \widehat{U(1)}$ representations are characterized by the eigenvalues of the $\alpha_{0}^{(i)}$, zero modes of the chiral currents, which span a two-dimensional lattice $\Gamma$; each lattice represents a single theory. We can choose a particular class of lattices whose filling fractions match the Jain series and whose representations allow the previously mentioned extension of the symmetry $\widehat{U(1)} \times \widehat{U(1)} \rightarrow \widehat{U(1)})_{\text {diagonal }} \times \widehat{S U(2)_{1}}$.5.

The corresponding spectrum of quasi-particles with $\nu=2 /(2 p+1)$ is found to be [5]:

$$
\begin{aligned}
Q & =\frac{n_{1}+n_{2}}{2 p+1} \quad n_{1}, n_{2} \in \mathbf{Z} \text { and } p=2,4, \ldots, \\
h & =\frac{1}{2} \frac{\theta}{\pi}=\frac{1}{2}\left(n_{1}^{2}+n_{2}^{2}\right)-\frac{1}{2(2 p+1)}\left(n_{1}+n_{2}\right)^{2} \quad n_{1}, n_{2} \in \mathbf{Z} .
\end{aligned}
$$

With a simple change of variables, this spectrum can be decomposed into charged and neutral sectors. The new integer quantum numbers $l$ and $n$ are defined by $n_{1}+n_{2}=2 l+\alpha$ and $n_{1}-n_{2}=2 n+\alpha$, with $\alpha=0,1$; the spectrum of charges and quantum statistics is divided into two parts with "parity " $\alpha$ 9

-Type $I: \alpha=0$ 


$$
\begin{aligned}
Q & =\frac{2 l}{2 p+1} \quad l \in \mathbf{Z} \text { and } p=2,4, . ., \\
h & =\frac{1}{2} \frac{\theta}{\pi}=\frac{1}{2 p+1} l^{2}+n^{2} \quad l, n \in \mathbf{Z} .
\end{aligned}
$$

-Type II : $\alpha=1$

$$
\begin{aligned}
Q & =\frac{2}{2 p+1}\left(l+\frac{1}{2}\right) \quad ; l \in \mathbf{Z} \text { and } p=2,4, \ldots, \\
h & =\frac{1}{2} \frac{\theta}{\pi}=\frac{1}{2 p+1}\left(l+\frac{1}{2}\right)^{2}+\frac{(2 n+1)^{2}}{4} \quad l, n \in \mathbf{Z} .
\end{aligned}
$$

After this change of variables, the neutral and charged excitations are indeed factorized.

The number $l$ counts the units of fractional charge and the number $n$ labels the neutral quasi-particles. In this context, $p$ has a clear meaning related to the compactification radius of the field $\left(\varphi^{(1)}+\varphi^{(2)}\right)$, which represents the charged sector of the theory $\left.(\widehat{U(1)})_{\text {diagonal }}\right)$.

It is useful to introduce the currents and the Virasoro generators whose eigenvalues are labelled by the new quantum numbers $l$ and $m$ :

$$
\begin{array}{lll}
J=J^{(1)}+J^{(2)}, & \frac{J_{0}}{\sqrt{2 p+1}} & \longrightarrow Q=\frac{2 \ell}{2 p+1} \\
J^{3}=\frac{1}{2}\left(J^{(1)}-J^{(2)}\right), & J_{0}^{3} & \longrightarrow n ; \\
L=L^{(1)}+L^{(2)}=L^{Q}+L^{S}, & L_{0} & \longrightarrow \frac{\ell^{2}}{2 p+1}+n^{2} ; \\
L^{Q}=\frac{1}{4}:(J)^{2}:, \quad L^{S}=:\left(J^{3}\right)^{2}: & &
\end{array}
$$

Each value of the spectrum corresponds to a highest weight state of the Abelian current algebra $\widehat{U(1)} \times \widehat{U(1)}$; each highest weight state has an infinite tower of states on top generated by the action of $\alpha_{n}^{(i)}, n<0, i=1,2$.

Up to now we did not make explicit the extended symmetry $\widehat{U(1)} \times$ $\widehat{S U(m)_{1}}$. The natural way to proceed is to incorporate two additional chiral currents of dimension one [10]:

$$
J^{ \pm}=: \exp ( \pm i \sqrt{2} \varphi):
$$


with $\varphi=\frac{1}{2}\left(\varphi^{(1)}-\varphi^{(2)}\right)$. Computing the commutators of the Fourier modes of $J^{ \pm}, J^{3}$, we get

$$
\begin{aligned}
& {\left[J_{n}^{a}, J_{m}^{b}\right]=i \epsilon^{a b c} J_{n+m}^{c}+\frac{k}{2} \delta^{a b} \delta_{n+m, 0}, \quad k=1, a, b, c=1,2,3} \\
& {\left[L_{n}^{S}, J_{m}^{a}\right]=-m J_{n+m}^{a}}
\end{aligned}
$$

which is the $S U(2)_{1}$ current algebra. Therefore, it is clear that the extended symmetry is a consequence of the choice of the particular lattice $\Gamma$.

So far, we described the Hall fluid with $\nu=\frac{2}{2 p+1}$ in terms of the rational conformal field theory $\left.\widehat{U(1)})_{\text {diagonal }} \times \widehat{S U(2)}\right)_{1}$. Next, we compare this theory with the alternative minimal models of the $W_{1+\infty}$ algebra [9]. A detailed description of the incompressible fluids by $w_{\infty}$ transformations and the definitions of the $W_{1+\infty}$ algebra is given in [6]. Here, we just summarize the main properties which are useful in our analysis.

In general, the $c=m W_{1+\infty}$ unitary representations are of two types: generic or degenerate. The generic $W_{1+\infty}$ theories are equivalent to chiralboson theories; these have the same spectra of filling fractions, fractional charge and statistic of excitations. For the $c=2$ case the representations of the Abelian algebra $\widehat{U(1)} \times \widehat{U(1)}$ with charges $\left(n^{1}, n^{2}\right)$ (eigenvalues of $J_{0}^{1}$ and $\left.J_{0}^{2}\right)$ are one-to-one equivalent to those of the $W_{1+\infty}$ algebra satisfying $\left(n^{1}-n^{2}\right) \notin Z$. On the other hand, the degenerate $W_{1+\infty}$ representations are not equivalent to the Abelian ones, and are contained into them, their charges $\left(n^{1}, n^{2}\right)$ satisfy $\left(n^{1}-n^{2}\right) \in Z$. A remarkable property of the degenerate representations is that they correspond one-to-one to the hierarchical plateaus and are thus physically relevant [6]. The $c=m W_{1+\infty}$ degenerate representations are equivalent to the representations of the $\widehat{U(1)} \times \mathcal{W}_{m}$ algebra, where $\mathcal{W}_{m}$ is the Zamolodchikov-Fateev-Lukyanov algebra at $c=m-1$ [11. The $S U(m)$ quantum number associated with the neutral sector we mentioned before arises from the fusion rules of the $\mathcal{W}_{m}$ algebra, which are isomorphic to the tensor product of the $S U(m)$ Lie algebra representations.

In the $c=2$ case [9], we have

$$
\text { degenerate } W_{1+\infty} \text { reps }=\widehat{U(1)} \times \text { Vir reps }
$$

where the $\widehat{U(1)}$ Abelian algebra corresponds to the charged sector and the $\mathcal{W}_{2} \approx$ Virasoro algebra accounts for the neutral one. 
The spectrum of the $W_{1+\infty}$ minimal models is the same as that of the $\widehat{U(1)})_{\text {diagonal }} \times \widehat{S U(2)_{1}}$ theories (5⿹丁口), with the integer $n$ restricted to $n=$ $0,1,2, \ldots$ : each point of the lattice defined by the numbers $n, l$, corresponds to one degenerate $W_{1+\infty}$ representation, while in the $\left.\widehat{U(1)} \times S \widehat{U U(2)}\right)_{1}$ this is a $\widehat{U(1)} \times \widehat{U(1)}$ representation with the same Virasoro weight.

Using the characters of the $\widehat{S U(2)})_{1}$ representations with isospin $\sigma=\alpha / 2=$ $0,1 / 2$ and the Virasoro representations $h=n^{2} / 4$ [12] [10] it is possible to count the number of edge excitations in the neutral sector, which is different in the two theories [9]. Their expressions are:

$$
\begin{aligned}
\chi_{\sigma=0}^{\widehat{S U(2)})_{1}} & =\frac{1}{\eta(q)^{3}} \sum_{k \in \mathbf{Z}}(6 k+1) q^{(6 k+1)^{2} / 12}, \\
\chi_{\sigma=1 / 2}^{S(2)_{1}} & =\frac{1}{\eta(q)^{3}} \sum_{k \in \mathbf{Z}}(6 k+2) q^{(6 k+2)^{2} / 12}, \\
\chi_{L_{0}^{S}=n^{2} / 4}^{\mathrm{Vir}} & =\frac{q^{n^{2} / 4}\left(1-q^{n+1}\right)}{\eta(q)}=\frac{q^{n^{2} / 4}-q^{(n+2)^{2} / 4}}{\eta(q)} .
\end{aligned}
$$

It is convenient to relate them to the characters of $\widehat{U(1)}$ representations

$$
\chi_{L_{0}=h}^{\widehat{U(1)}}=\frac{q^{h}}{\eta(q)}, \quad \eta(q)=q^{1 / 24} \prod_{k=1}^{\infty}\left(1-q^{k}\right),
$$

as follows:

$$
\begin{aligned}
\chi_{\sigma=0}^{S \widehat{U(2)})_{1}} & =\frac{1}{\eta(q)} \sum_{k \in \mathbf{Z}} q^{k^{2}}, \quad \chi_{\sigma=1 / 2}^{S \widehat{U(2)})_{1}}=\frac{1}{\eta(q)} \sum_{k \in \mathbf{Z}} q^{(2 k+1)^{2} / 4}, \\
\widehat{\chi_{J_{0}^{3}=n / 2}} & =\sum_{\ell=0}^{\infty} \chi_{L_{0}^{S}=(n+2 \ell)^{2} / 4}^{\mathrm{Vir}} .
\end{aligned}
$$

From these formulae, the characters of the two theories are related as follows [7]:

$$
\begin{aligned}
& \chi_{\sigma=0}^{\widehat{S U(2)})_{1}}=\sum_{k=0}^{\infty}(2 k+1) \chi_{L_{0}^{S}=(2 k)^{2} / 4}^{\mathrm{Vir}}, \\
& \chi_{\sigma=1 / 2}^{\widehat{S U(2)}}=\sum_{k=0}^{\infty}(2 k+2) \chi_{L_{0}^{S}=(2 k+1)^{2} / 4}^{\mathrm{Vir}} .
\end{aligned}
$$


The above relations correspond to the following decomposition of $\widehat{S U(2)})_{1}$ representations into $\widehat{U(1)}$ and Virasoro ones:

$$
\begin{aligned}
& \{\sigma=0\}_{S \widehat{U(2)} 1}=\sum_{k \in \mathbf{Z} \text { even }}\left\{J_{0}^{3}=\frac{k}{2}\right\}_{\widehat{U(1)}}=\sum_{s=0}^{\infty}(2 s+1)\left\{L_{0}^{S}=s^{2}\right\}_{\mathrm{Vir}}, \\
& \left\{\sigma=\frac{1}{2}\right\}_{\widehat{S U(2)})_{1}}=\sum_{k \in \mathbf{Z} \text { odd }}\left\{J_{0}^{3}=\frac{k}{2}\right\}_{\widehat{U(1)}}=\sum_{s=1 / 2,}^{\infty} \sum_{s \in \mathbf{Z}^{+}+1 / 2}(2 s+1)\left\{L_{0}^{S}=s^{2}\right\}_{\mathrm{Vir}} .
\end{aligned}
$$

These expressions show: first, that the two $\widehat{S U(2)}{ }_{1}$ representations sum up the neutral spectrum of the Abelian theory in the two sectors, type I and II, for $\sigma=0$ and $1 / 2$ respectively; second, that the multiplicities of the decomposition into Virasoro representations match the familiar multiplicities $(2 s+1)$ of the $S U(2)$ Lie algebra representations. We see that the minimal models contains the Virasoro $h=s^{2}$ representations with multiplicity 1 , while the $S U(2)_{1}$ theory contains $(2 s+1)$ of them. Therefore, one can obtain the minimal models from the $\widehat{U(1)} \times S \widehat{U(m)_{1}}$ theory by selecting one state for each $S U(2)$ multiplet: this can be done by imposing the constraint on the states 9]:

$$
\left.J_{0}^{-} \mid \text {minimal state }\right\rangle=0
$$

The $\widehat{U(1)} \times S \widehat{U(m)}$, states which satisfy the constraint belong to the minimal models, and the others are projected out. Therefore, the $W_{1+\infty}$ minimal models are defined by the $\widehat{U(1)} \times S \widehat{S(m})_{1}$ Hilbert space plus the constraint (17). The evident consequence of the incorporation of the constraint is the breaking of the $S U(2)$ symmetry and the fixing of the $U(1)$ quantum number $m=-s$.

The Hamiltonian approach of Ref [9] is an equivalent way to present the results we just described. First, let us consider the standard $\widehat{U(1)} \times \widehat{U(1)}$ Hamiltonian

$$
H=\frac{1}{R}\left(v L_{0}^{Q}+v^{\prime} L_{0}^{S}-\frac{1}{12}\right)
$$

Next, we add a (non-local) relevant term:

$$
\Delta H=\gamma J_{0}^{+} J_{0}^{-} \quad \gamma \in[0, \infty)
$$


This is diagonal in the previous $\left(m, s^{2}\right)$ basis, and assigns the energy $\gamma[m(m+$ $1)-s(s+1)$ ] to each state of the $S U(2)$ multiplets. The coupling constant $\gamma$ has dimension of a mass which assures the relevancy of the term in the renormalization-group sense. For $\gamma \rightarrow \infty$, it selects the lowest weight in each $S U(2)$ multiplet, $m=-s$, in other words, implements the constraint [9].

Therefore, the infra-red limit of the theory defined by the Hamiltonian $H+\Delta H$ is the $c=2 W_{1+\infty}$ minimal model. This infra-red fixed point is known exactly and is conformally invariant because $\left[J_{0}^{+} \quad J_{0}^{-}, L_{0}^{S}\right]=0$.The trajectory in the theory space defined by the action of the renormalizationgroup interpolates between the $\widehat{U(1)} \times \widehat{S U(2)}$, theory $(\gamma \rightarrow 0)$ and the $c=2$ minimal model $(\gamma \rightarrow \infty)$. The fact that the conformal field theories are describing the low energy edge excitations, makes physically significant this infra-red limit, which is reached without any fine-tuning.

\section{$3 c=m \mathbf{W}_{1+\infty}$ Minimal Models and $\widehat{U(1)} \times$ $S \widehat{U(m)})_{1}$ Theories}

The purpose of this paper is to generalize the previous analysis to describe the $c=m$ case. In particular, we will study the reduction in the number of the degrees of freedom from the $\widehat{U(1)} \times S \widehat{U(m)}$, to the $c=m W_{1+\infty}$ minimal models. We start with the algebraic analysis of the corresponding characters, and we complete the description including the Hamiltonian point of view.

As we mentioned in the Introduction, the $\widehat{U(1)} \times S \widehat{U(m)_{1}}$ theory can be built out of $m$ chiral bosons defined on the edge of the Hall sample. The zero modes of the chiral currents $J^{(i)}, i=1, \ldots m$, associated with these bosons, span a $m$-dimensional lattice. From the set of possible lattices, we choose the particular class, in which the filling fractions correspond to the Jain series $\nu=m /(m p+1)$. These lattices imply the symmetry extension from $\left.\widehat{U(1)}^{m} \rightarrow \widehat{U(1)} \times \widehat{S U(m)}\right)_{1}$.

The spectrum of the theory is given by [5]:

$$
Q=\frac{1}{p m+1} \sum_{i=1}^{m} n_{i}
$$




$$
2 h=\frac{\theta}{\pi}=\sum_{i=1}^{m} n_{i}^{2}-\frac{p}{m p+1}\left(\sum_{i=1}^{m} n_{i}\right)^{2} \quad n_{i} \in \mathbf{Z} .
$$

In the lattice $\Gamma$ the norm of the vectors is $h$, the total Virasoro dimension, and each point identifies a highest weight representation of $\widehat{U(1)}^{m}$. In order to perform the factorization into charged and neutral sectors, we introduce a set of definitions related with the $S U(m)$ Lie Algebra. Consider the $(m-1)$ dimensional sub-lattice $P$ generated by the fundamental weight $\Lambda^{i}$ of $S U(m)$ and $\boldsymbol{\Lambda}=\sum_{i=1}^{m-1} l_{i} \boldsymbol{\Lambda}^{i} \in P$ a vector of $P$. A standard basis for the weights is [13]:

$$
\begin{aligned}
& \mathbf{u}^{1}=\left(\frac{1}{\sqrt{2}}, \frac{1}{\sqrt{6}}, \frac{1}{\sqrt{12}}, \ldots \ldots . ., \frac{1}{\sqrt{k(k-1)}}, \ldots . ., \frac{1}{\sqrt{m(m-1)}}\right) \\
& \mathbf{u}^{2}=\left(-\frac{1}{\sqrt{2}}, \frac{1}{\sqrt{6}}, \ldots \ldots \ldots \ldots \ldots \ldots \ldots \ldots \ldots \ldots . . \frac{1}{\sqrt{m(m-1)}}\right) \\
& \cdot \\
& \mathbf{u}^{k}=\left(0, \ldots \ldots, 0, \ldots \ldots \ldots \ldots,-\frac{k-1}{\sqrt{k(k-1)}}, \ldots ., \frac{1}{\sqrt{m(m-1)}}\right) \\
& \mathbf{u}^{m}=\left(0, \ldots \ldots, 0, \ldots \ldots \ldots \ldots \ldots \ldots \ldots \ldots \ldots, 0, \frac{1}{\sqrt{m(m-1)}}\right)
\end{aligned}
$$

with

$$
\boldsymbol{\Lambda}^{i}=\sum_{j=1}^{i} \mathbf{u}^{j}
$$

The simple positive roots $\boldsymbol{\alpha}_{i}$ dual to the $\boldsymbol{\Lambda}^{i}$ are given by

$$
\boldsymbol{\alpha}_{i}=\mathbf{u}^{i}-\mathbf{u}^{i+1}
$$

with

$$
\boldsymbol{\Lambda}^{i} \cdot \boldsymbol{\alpha}_{j}=\delta_{j}^{i}, \quad \boldsymbol{\Lambda}^{i} \cdot \boldsymbol{\Lambda}^{j}=i-\frac{i j}{m} \quad i \leq j,
$$

and a vector in the root lattice is of the form $\boldsymbol{\gamma}=\sum_{j=1}^{i} k_{i} \boldsymbol{\alpha}_{i}$. The relation between the root lattice and the weight lattice is given by the Cartan matrix:

$$
\boldsymbol{\alpha}_{i}=2 \boldsymbol{\Lambda}^{i}-\boldsymbol{\Lambda}^{i-1}-\boldsymbol{\Lambda}^{i+1}
$$

The representations of $S U(m)$ Lie algebra can be characterized by a positive highest weight vector $\Lambda \in P^{+}$, in other words, the representations are labelled by $\left(l_{1}, \ldots, l_{m-1}\right)$ with $l_{i} \geq 0$. In addition, their $m$-ality is given by: 


$$
\alpha=\sum_{i=1}^{m-1} i l_{i} \bmod (m)
$$

$\alpha=0,1, \ldots m-1$.

Next, it is useful to introduce the following property

$$
\sum_{i=1}^{m-1} i l_{i}=\sum_{i=1}^{m-1} i\left(l_{i}+2 k_{i}-k_{i-1}-k_{i+1}\right) \quad \bmod (m),
$$

which means that the $m$-ality is invariant under the addition of a vector in the root lattice. We will denote $P_{\alpha}$ the $m$ sub-lattices of $P$ with the given value of $\alpha$ of the $m$-ality. Now, the factorization into charged and neutral sectors is obtained by the change of basis :

$$
\left(n_{1}, \ldots n_{m}\right) \rightarrow\left(l, l_{1}, \ldots, l_{m-1}\right)
$$

with $l=\sum_{i=1}^{m} n_{i}$ and $l_{i}=n_{i}-n_{i-1}$. The spectrum (20) can be rewritten:

$$
\begin{gathered}
Q=\frac{l}{p m+1}, \\
2 h=\frac{\theta}{\pi}=h_{\boldsymbol{\Lambda}}+\frac{l^{2}}{2 m(m p+1)} ; \\
h_{\boldsymbol{\Lambda}}=\frac{1}{2} \sum_{i=1}^{m-1}\left(\frac{i m-i^{2}}{m}\right) l_{i}^{2}+\sum_{j=1}^{m-1} \sum_{i=1}^{j-1}\left(i-\frac{i j}{m}\right) l_{i} l_{j}=\frac{1}{2}|\boldsymbol{\Lambda}|^{2} .
\end{gathered}
$$

The integer $l$ identifies the charged sector and the $l_{i}$ the neutral one.

For the purpose of comparison of the $\widehat{S U(m)}{ }_{1}$ and $\mathcal{W}_{m}$ neutral spectrum, the last expression is not yet completely appropriate; it is convenient to consider an $\alpha$-dependent change of basis, making explicitly the $m$-ality of the theory. We modify the previous transformation, (taking into account the property (26)) as follows:

For $\alpha=\alpha_{j}, j=0,1, \ldots, m-1$ :

$$
m l+\alpha_{j}=\sum_{i=1}^{m} n_{i}
$$




$$
l_{i}=n_{i}-n_{i-1}=2 k_{i}-k_{i-1}-k_{i+1}+l_{i}^{\prime}, \quad 1 \leq i \leq m-1 ;,
$$

where $l_{i}^{\prime}=1$ if $i=j$ or $l_{i}^{\prime}=0$ if $i \neq j$. Here, $\alpha_{i}=i$ with $i=0, \ldots, m-1$.

The spectrum in the new basis is given by:

For $\alpha=\alpha_{j}$ :

$$
\begin{aligned}
Q_{\alpha_{j}} & =\frac{m l+\alpha_{j}}{p m+1} \\
2 h_{\alpha_{j}} & =\frac{\theta}{\pi}=h_{\boldsymbol{\Lambda}}+\frac{\left(m l+\alpha_{j}\right)^{2}}{2 m(m p+1)},
\end{aligned}
$$

with:

$$
h_{\boldsymbol{\Lambda}}=\frac{1}{2} \sum_{i=1}^{m-1}\left(\frac{i m-i^{2}}{m}\right) l_{i}^{2}+\sum_{j=1}^{m-1} \sum_{i=1}^{j-1}\left(i-\frac{i j}{m}\right) l_{i} l_{j},
$$

where $\boldsymbol{\Lambda}=\left(l_{1}, \ldots, l_{m-1}\right)$, with $l_{i}$ given by the previous expression (30).

We introduce the $S U(m)_{1}$ representations corresponding to $\boldsymbol{\Lambda}_{S U(m)_{1}}=$ $\left(l_{1}, l_{2}, \ldots ., l_{m-1}\right)$ with $\sum_{i=1}^{m-1} l_{i} \leq 1$, and define $\boldsymbol{\Lambda}_{\alpha_{i}}=(0, \ldots .1, \ldots 0)$, with the $i$ th component equal to 1 and $\boldsymbol{\Lambda}_{\alpha_{0}}=(0, \ldots 0, \ldots .0)$. This notation allows to rewrite the previous change of basis in the following compact way:

For $\alpha=\alpha_{j}$ :

$$
\begin{aligned}
m l+\alpha_{j} & =\sum_{i=1}^{m} n_{i}, \\
\boldsymbol{\Lambda} & =\boldsymbol{\Lambda}_{\alpha_{j}}+\gamma ;
\end{aligned}
$$

Now, we are ready to compare the $\widehat{U(1)} \times S \widehat{S(m})_{1}$ theory with the $c=$ $m W_{1+\infty}$ minimal models and study the reduction in the number of the degrees of freedom. The characters of the representations provide the relevant information as in the previous section, because the projection from $\widehat{U(1)} \times$ $\widehat{S U(m)}$, to the minimal models can be obtained from the decomposition of the characters of $\widehat{S U(m)})_{1}$. The $\left.\widehat{S U(m)}\right)_{1}$ character for the $\boldsymbol{\Lambda}_{\alpha_{j}}$ representation is given by [12]: 


$$
\chi_{\boldsymbol{\Lambda}_{\alpha_{j}}}^{S \widehat{U(m)})_{1}}=\frac{1}{\eta^{m^{2}-1}} \sum_{k_{i}=-\infty}^{\infty} \operatorname{dim}\left(\boldsymbol{\Lambda}_{\alpha_{j}}+(m+1) \boldsymbol{\gamma}\right)\left(q^{\frac{i}{2 m-1}\left|\vec{p} \boldsymbol{\Lambda}_{\alpha_{j}}+(m+1) \boldsymbol{\gamma}\right|^{2}}\right)
$$

with

$$
\vec{p} \boldsymbol{\Lambda}_{\alpha_{j}}=\sum_{i=1}^{m-1} \Lambda_{i}+\Lambda_{\alpha_{j}} ; \gamma=\sum_{i=1}^{m-1} k_{i} \alpha_{i} \text {, a vector of the root lattice and }
$$

$\operatorname{dim}\left(\boldsymbol{\Lambda}_{\alpha_{i}}+(m+1) \boldsymbol{\gamma}\right)$ the dimension of the $S U(m)$ Lie Group representations given by:

$$
\begin{aligned}
& \operatorname{dim}\left(l_{1}, \ldots, l_{m-1}\right)=\left(1+l_{1}\right) \ldots \ldots \ldots \ldots \ldots \ldots \ldots \ldots . .\left(1+l_{m-1}\right) \\
& \times\left(1+\frac{l_{1}+l_{2}}{2}\right) \ldots \ldots \ldots\left(1+\frac{l_{m-2}+l_{m-1}}{2}\right) \\
& \times \ldots \times\left(1+\frac{l_{1}+\ldots+l_{m-1}}{m-1}\right) \\
& =\prod_{j=1}^{m-1}\left(\prod_{k=1}^{m-j}\left(1+\sum_{i=k}^{j+k+1} \frac{l_{i}}{j}\right)\right)
\end{aligned}
$$

For the minimal models we consider the characters of the degenerate $\mathcal{W}_{m}$ representations [14]:

$$
\begin{aligned}
\chi_{h_{\Lambda}}^{\mathcal{W}_{m}}= & \frac{q^{h_{\Lambda}}}{\eta^{m-1}}\left(1-q^{l_{1}+1}\right)\left(1-q^{l_{2}+1}\right) \ldots\left(1-q^{l_{m-1}+1}\right) \\
& \times\left(1-q^{l_{1}+l_{2}+2}\right)\left(1-q^{l_{2}+l_{3}+2}\right) \ldots \\
& \times \ldots \\
& \times\left(1-q^{l_{1}+l_{2}+\ldots+l_{m-1}+m-1}\right) .
\end{aligned}
$$

For both characters, (34) and (37), it is possible to establish a relation with the characters of $\widehat{U(1)}{ }^{m-1}$ representations, as follows:

$$
\chi_{\mathbf{\Lambda}_{\alpha j}}^{S U(m)_{1}}=\frac{1}{\eta^{m-1}} \sum_{k_{i} \in \mathbf{Z}} q^{h_{\Lambda}}
$$

where we consider the value of $h_{\Lambda}$ (32) which corresponds to the sector $\alpha_{j}$.

Finally, as in the $c=2$ case, we can relate the characters of the two theories in a direct way: 


$$
\chi_{\boldsymbol{\Lambda}_{\alpha j}}^{\widehat{S U(m})_{1}}=\sum_{k_{i} \in \Phi} \operatorname{dim}\left(\boldsymbol{\Lambda}_{\alpha_{i}}+\gamma\right) \chi_{h_{\Lambda}}^{\mathcal{W}_{m}}
$$

with $\Phi=\left\{k_{i} / 2 k_{i}-k_{i-1}-k_{i+1}+l_{i}^{\prime} \geq 0\right\}$ and as in (38) $h_{\Lambda}$ given in (32) which corresponds to the sector $\alpha_{j}$.

The two last identities have been checked with the help of the program Mathematica [15 for $m=3,4,5$ by an expansion in powers of $q$. We find for the general $c=m$ theories the following identities:

$$
\left\{\Lambda_{\alpha_{j}}\right\}_{S U(m)_{1}}=\sum_{k_{i} \in \mathbf{Z}}\left\{h=h_{\boldsymbol{\Lambda}}\right\}_{U\left(\widehat{1)^{m}}-1\right.}=\sum_{k_{i} \in \Phi} \operatorname{dim}_{S U(m)}(\boldsymbol{\Lambda})\left\{L_{0}=h_{\boldsymbol{\Lambda}}\right\}_{\mathcal{W}_{m}}
$$

We conclude again that the minimal models contains the $h_{\boldsymbol{\Lambda}}$ representations with multiplicity 1 , while the $S U(m)_{1}$ contains $\operatorname{dim}_{S U(m)}(\boldsymbol{\Lambda})$ of them.

To complete our analysis, we consider the Hamiltonian of the $c=m$ $W_{1+\infty}$ minimal models, which is completely analogous to the $c=2$ case. The projection from the Abelian theory to the minimal models is obtained by the application of the constraint on the states given by

$$
\left.E_{0}^{-\boldsymbol{\alpha}_{i}} \mid \text { minimal state }\right\rangle=0 ;,
$$

with $i=1, \ldots, m-1$ and the $E_{0}^{-\alpha_{i}}$ the ladder operators of the $S U(m)$ Lie algebra. The analogous to $E_{0}^{-\boldsymbol{\alpha}_{i}}$ in the $S U(2)$ case is $J_{0}^{-}$, which select one state of the multiplet -the lowest weight state. The relevant term we have to add to the Hamiltonian to accomplish the projection has the form:

$$
\Delta H=\gamma \sum_{i=1}^{m-1}\left(E_{0}^{\boldsymbol{\alpha}_{i}} E_{0}^{-\boldsymbol{\alpha}_{i}}\right),
$$

because this is the unique term which annihilates the $S U(m)$ lowest-weight state and is diagonal in the $S \widehat{U(m})_{1}$ basis. As before, the infra-red limit $\gamma \rightarrow \infty$ corresponds to the minimal models, because other states in the hyper multiplets acquire an energy proportional to $\gamma$. The Hamiltonian description, gives us a physical framework in which it is possible to discuss the differences between the two types of theories. The renormalization group action shows a natural link between the theories relating the limits $\gamma \rightarrow 0$ and $\gamma \rightarrow \infty$, corresponding to the Abelian theory and the minimal models respectively. 


\section{Acknowledgements}

I would like to thank Guillermo Zemba, for suggesting me this problem, for discussions and for reading and commenting on the manuscript. I would also like to thank Andrea Cappelli for useful comments. I also enjoyed discussions with Horacio Casini.

This work was supported by a FOMEC fellowship of the Education Ministry of Argentina.

\section{References}

[1] For a review see: R. A. Prange and S. M. Girvin, The Quantum Hall Effect, Springer Verlag, New York A.

[2] For a review, see: X. G. Wen, Int. J. Mod. Phys. 6 B (1992) 1711; Adv. in Phys. 44 (1995) 405.

[3] For a review see: J. K. Jain, Adv. in Phys. 44 (1992) 105, Science 266 (1994) 1199.

[4] R. B. Laughlin, Phys. Rev. Lett. 50 (1983) 1395; for a review see: R. B. Laughlin, Elementary Theory: the Incompressible Quantum Fluid, in [1].

[5] J. Fröhlich and A. Zee, Nucl. Phys. 364 B (1991) 517; X.-G. Wen and A. Zee, Phys. Rev. 46 B (1993) 2290. J. Fröhlich and E. Thiran, J. Stat. Phys. 76 (1994) 209; J. Fröhlich, T. Kerler, U. M. Studer and E. Thiran, Nucl. Phys. B 453 (1995) 670.

[6] A. Cappelli, C. A. Trugenberger and G. R. Zemba, Nucl. Phys. 448 (1995) 470; for a review, see: Nucl. Phys. (Proc. Suppl.) B 45A (1996) 112.

[7] A.Cappelli, C. A. Trugenberger and G. R. Zemba, Nucl. Phys. 396 B (1993) 465; Phys. Lett. 306 B (1993) 100; Phys. Rev. Lett. 72 (1994) 1902; for a review, see: A.Cappelli, G.V.Dunne, C.A.Trugenberger and G.R.Zemba, Nucl. Phys. B (Proc. Suppl.) 33 C (1993) 21.(1990); 
[8] A. Cappelli, C. Mendez, J. M. Simonin and G. R. Zemba, preprint condmat/9806238, Phys. Rev. B in press.

[9] A. Cappelli and G.R. Zemba, hep-th 9808179, Nucl. Phys. B, in press.

[10] P. Di Francesco, P. Mathieu and D. Senechal, Conformal Field Theories, Springer-Verlag, (1996).

[11] V.A. Fateev and A.B. Zamolodchikov, Nucl. Phys. B 280 (1989) 644;

V.A. Fateev and S.L. Lukyanov, Int. J. Mod. Phys. A 3(1988) 507.

[12] C. Itzykson, Nucl. Phys. (Proc. Suppl.) 5 B (1988) 150.

[13] B. G. Wybourne, Classical Groups for Physicists, Wiley, New York (1974).

[14] V. Kac and A. Radul, Comm. Math. Phys. 157 (1993) 429; E. Frenkel, V. Kac, A. Radul and W. Wang, Comm. Math. Phys. 170 (1995) 337; H. Awata, M. Fukuma, Y. Matsuo and S. Odake, Prog. Theor. Phys. (Supp.) 118 (1995) 343.

[15] S. Wolfram, Mathematica, Addison-Wesley, New York (1991) 